SUBJECT AREAS:

CONDENSED-MATTER

PHYSICS

PHASE TRANSITIONS AND CRITICAL PHENOMENA

Received

3 September 2014

Accepted

5 February 2015

Published

9 March 2015

Correspondence and requests for materials should be addressed to T.C. (cuitian@jlu.edu.

\section{High-temperature Superconductivity in compressed Solid Silane}

\author{
Huadi Zhang, Xilian Jin, Yunzhou Lv, Quan Zhuang, Yunxian Liu, Qianqian Lv, Kuo Bao, Da Li, Bingbing Liu \\ \& Tian Cui
}

State Key Laboratory of Superhard Materials, College of physics, Jilin University, Changchun, 130012, P. R. China.

Crystal structures of silane have been extensively investigated using ab initio evolutionary simulation methods at high pressures. Two metallic structures with $P 2_{1} / c$ and $C 2 / \mathrm{m}$ symmetries are found stable above $383 \mathrm{GPa}$. The superconductivities of metallic phases are fully explored under BCS theory, including the reported $\mathrm{C2} / \mathrm{c}$ one. Perturbative linear-response calculations for $\mathrm{C2} / \mathrm{m}$ silane at $610 \mathrm{GPa}$ reveal a high superconducting critical temperature that beyond the order of $10^{2} \mathrm{~K}$.

- inding high temperature superconductor is one of the important issues in scientific fields. Since N. W. Ashcroft announced the prediction of the metallic hydrogen ${ }^{1}$, scientists began to explore the hydrogen superconductor. The research about it has never been stopped. Recent theoretical study showed that the $T_{c}$ of solid metallic hydrogen has achieved $100 \mathrm{~K}^{2}$. It is still a subject that is full of significant, despite the experimental metallic hydrogen had not found until pressure achieved $300 \mathrm{GPa}^{3,4}$.

The hydrogen-rich compounds, especially for the hydrides of group IV, are expected high temperature superconductor for comprehending the superconductivity of metallic hydrogen. This attracted more attention on hydrides of group IV. So far, the theoretical studies of $\mathrm{GeH}_{4}$ and $\mathrm{SnH}_{4}$ have predicted high superconductivities with maximal $T_{c}$ reaching $64 \mathrm{~K}$ at $220 \mathrm{GPa}$ for $\mathrm{GeH}_{4}{ }^{5}$ and $62 \mathrm{~K}$ at $200 \mathrm{GPa}$ for $\mathrm{SnH}_{4}{ }^{6}$. In addition, more extensive theoretical and experimental efforts have attempted to reveal the structures and superconductivity of silane. Feng et al. ${ }^{7}$ found a Pman structure with high superconducting transition temperature $T_{\mathrm{c}} 166 \mathrm{~K}$ at $202 \mathrm{GPa}$. Pickard and $\mathrm{Needs}^{8}$ also predicted the structures of $\mathrm{SiH}_{4}$ and studied the structural properties, mentioned the possibility of superconductivity in a $C 2 / c$ phase. Later Yao et al. ${ }^{9}$ showed that the Pman structure is in reality not stable by phonon calculations whereas a new $\mathrm{C} 2 / \mathrm{c}$ structure is dynamically stable and the superconductivity is close to $50 \mathrm{~K}$ at $125 \mathrm{GPa}$. Eremets et al..$^{10}$ reported that the metallization of silane occurs between 50 and $65 \mathrm{GPa}$ with the $P 6_{3}$ symmetry and the superconducting transition temperature of $17 \mathrm{~K}$ at 96 and $120 \mathrm{GPa}$ by using electrical resistance measurements. Chen et al. ${ }^{11}$ demonstrated that the experimental $P 6_{3}$ metallic structure is dynamically unstable by the phonon calculations, and a new Cmca phase was predicted. The Cmca silane with a layered network was considered the most likely candidate with the superconducting transition temperature in the range of 20-75 K. Later, Miguel Martinez-Canales et al. ${ }^{12}$ found the lower enthalpy structures by using the evolutionary algorithm USPEX than those found in previous studies, and confirmed that $\mathrm{SiH}_{4}$ is a low-temperature superconductor with a transition temperature of $17 \mathrm{~K}$. These published theoretical and experimental articles indicate that silane is one of the significant materials for comprehending the superconductivity in metallic hydrogen under high pressures. So far, the high-pressure structures of silane are still in discussion, and the expected hightemperature superconductivity similar with metallic hydrogen has not been reported. In this paper, the highpressure crystal structures and potential superconducting property of silane have been extensively explored. Two metallic structures with space groups $P 2_{1} / c$ and $C 2 / m$ have been found stable from 383 to $606 \mathrm{GPa}$ and above $606 \mathrm{GPa}$ respectively. Further calculations discover that the superconducting transition temperature has more than $100 \mathrm{~K}$ for the $C 2 / \mathrm{m}$ phase. In addition, the superconductivity of $C 2 / c^{8}$ has been deeply explored in our work.

\section{Results and Discussion}

The crystal structures of silane were predicted with one to six $\mathrm{SiH}_{4}$ formula units per cell. Several competitive candidates for energy with the space groups $C 2 / c$ (2 molecules/cell), Cmcm (2 molecules/cell), $14 / \mathrm{mmm}(3$ molecules/cell), $P 2_{1} / c$ (4 molecules/cell), $P 2_{1} / m$ (4 molecules/cell), $P-1$ (4 molecules/cell) and $C 2 / m$ (6 molecules/cell) were obtained. The enthalpies of candidates are plotted as a function of pressures in Figure 1 . Above the $300 \mathrm{GPa}$, there are two competitive enthalpy structures with the $C 2 / c$ and $P 2_{1} / c$ symmetry. The maximum enthalpy difference between them is only 0.01 (eV/unit cell) over the pressure range from $300 \mathrm{GPa}$ to 


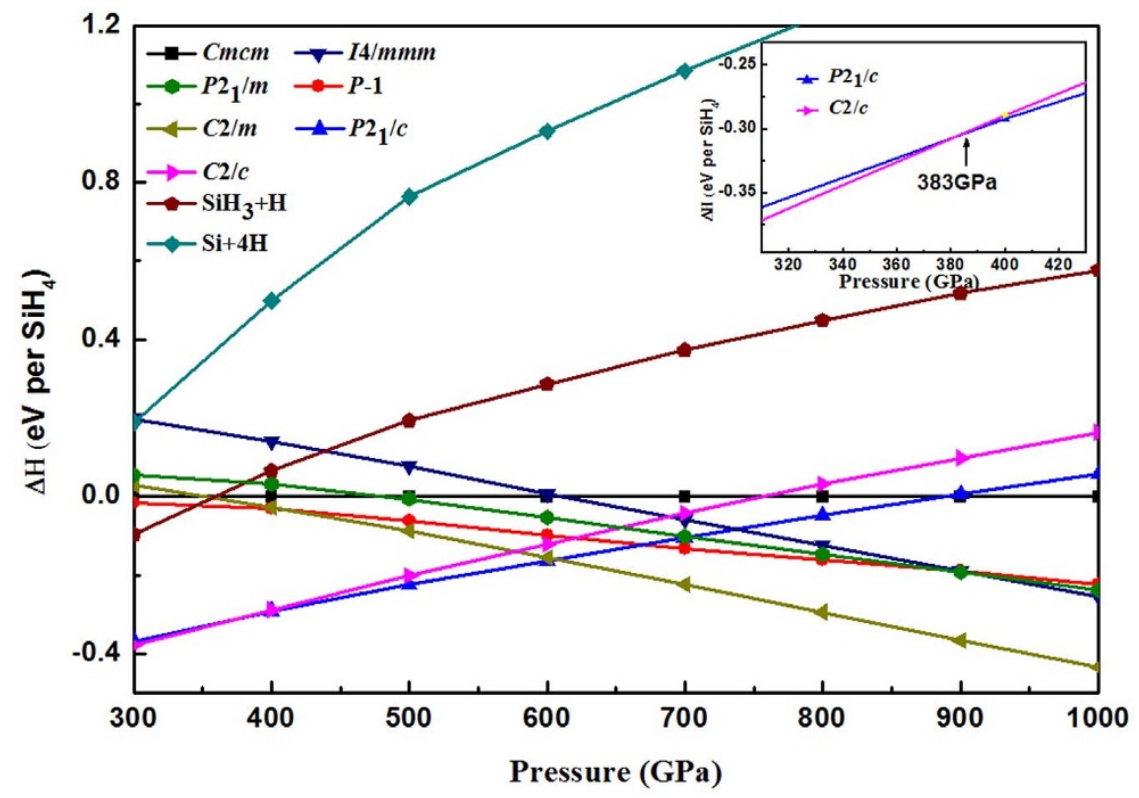

Figure 1 Enthalpy difference curves of Silane. Calculated enthalpies per $\mathrm{SiH}_{4}$ unit of various structures relative to our predicted $\mathrm{Cmcm}$ structure as a function of pressure range from 300-1000 GPa. Inset: Enthalpies in the pressure range from $310 \mathrm{GPa}$ to $430 \mathrm{GPa}$.

$383 \mathrm{GPa}$, see Figure 1 inset. The $C 2 / \mathrm{c}$ phase predicted in this work is identical with the one proposed by C. J. Pickard and R. J. Needs ${ }^{8}$, which forms three-dimensional networks. Above $383 \mathrm{GPa}, P 2_{1} / c$ phase take over $C 2 / c$ phase and becomes most competitive on enthalpy. As shown in Figure 2(a), Si atoms show a fold layered arrangement with $\mathrm{H}$ atoms site around them. There are four $\mathrm{SiH}_{4}$ units with five unequivalent atoms in the conventional cell. One $\mathrm{Si}$ atom and four $\mathrm{H}$ atoms occupy the crystallographic $4 e$ position with 1 symmetry in this monoclinic crystal. The shortest distance between $\mathrm{H}$ atoms is $1.045 \AA$ at $400 \mathrm{GPa}$, which is longer than the $0.762 \AA$ of the $\mathrm{H}-\mathrm{H}$ bond length in " $\mathrm{H}_{2}$ " unit in the $\mathrm{Cmca}-12$ structure ${ }^{13}$ of solid hydrogen at the same pressure. $P 2_{1} / c$ phase keep stable on enthalpy at least to $606 \mathrm{GPa}$ until another competitive phase with $C 2 / m$ symmetry appears, as shown in Figure $1 . C 2 / m$ phase obtains monoclinic base-centered lattice, and contains six formula units in the conventional cell. There are three unequivalent $\mathrm{Si}$ atoms occupy the $4 i$ position with $\mathrm{m}$ symmetry, whereas nine $\mathrm{H}$ atoms sit on the $8 j$ (site symmetry is 1 ), $4 i$ (site symmetry is $\mathrm{m}$ ) and $4 g$ (site symmetry is 2 ) positions respectively. The shortest distance between two $\mathrm{H}$ atoms is $1.009 \AA$, and slightly shorter than $1.045 \AA$ in the $P 2_{1} / c$ phase. The coordination of $\mathrm{Si}$ atoms in $C 2 / c, P 2_{1} / \mathrm{c}$, and $\mathrm{C} 2 / \mathrm{m}$ are all eleven, i.e. each $\mathrm{Si}$ atom bond with eleven $\mathrm{H}$ atoms, which is quite different with the molecule crystal under low pressure ${ }^{14}$ and implies some different

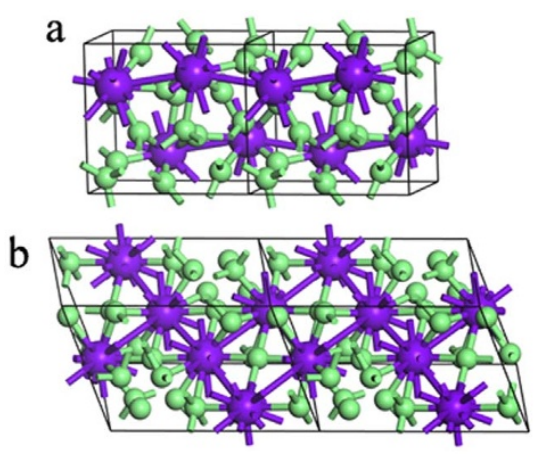

Figure $2 \mid$ Structures of silane. Big atoms depict Si, while small atoms represent $\mathrm{H}$. (a) Conventional cell of $P 2_{1} / c$ silane, stable between 383 and $606 \mathrm{GPa}$. (b) Primitive cell of $C 2 / m$ silane, favored above $606 \mathrm{GPa}$. physical characters. Detail parameters of the structures are listed in Table 1. The decomposition enthalpies reference to $\mathrm{Cmca}-12^{13}$ structure of $\mathrm{H}_{2}$ (below $500 \mathrm{GPa}$ ), I4 $/$ /amd $d^{13,15}$ structure of $\mathrm{H}_{2}$ (500$1000 \mathrm{GPa}), \mathrm{Fm}-3 \mathrm{~m}^{16}$ structure of $\mathrm{Si}$ and $\mathrm{C} 2 / \mathrm{c}^{17}$ structure of $\mathrm{SiH}_{3}$ can also be seen in Figure 1 (The comparison with $\mathrm{SiH}_{4}\left(\mathrm{H}_{2}\right)_{2}$ are shown in supplementary information). It is noteworthy that the enthalpies of the three structures are lower. Moreover, $P 2_{1} / c$ and $\mathrm{C} 2 / \mathrm{m}$ structures are found to be energetically much superior to previous structures ${ }^{7-9,11,12}$. Therefore, three monoclinic $\left(C 2 / c, P 2_{1} / c\right.$, and $\mathrm{C} 2 / \mathrm{m}$ ) phases can be taken as energetically stable structures of silane under high pressure range, see Figure 1.

The mechanical stability of structure can provide insight into the stability of materials. To evaluate the mechanical stability of the $C 2 / c$, $P 2_{1} / c$ and $C 2 / m$ phases, elastic constants have been calculated and listed in Table 2. According to the mechanical stability criteria, the crystal deformation energy is positive, this means the determinants of elastic constants matrix $C_{i j}$ should be positive ${ }^{18}$. Considering the crystal symmetry, the mechanical stability of expression will be further simplified ${ }^{19}$. It can be found that the elastic constants of these three structures satisfy the mechanical stability criteria, indicating that these three structures are mechanically stable. The phonon band structure and projected phonon density of states (PHDOS) of the three phases at selected pressures are presented in Figure 3. Absence of any imaginary frequency in the Brillouin zone establishes the dynamical stability. The PHDOS of these three structures shows that the heavier Si atoms dominate the low-frequency vibrations, and the lighter $\mathrm{H}$ atoms contribute significantly to the high-frequency modes.

To analysis the electronic properties of $C 2 / c, P 2_{1} / c$ and $C 2 / m$ phases, we first calculated the electronic density of states (DOS). As shown in Figure 4, they are all metals with large total DOS at Fermi level. Specially, for $C 2 / m$ structure, the total DOS of Fermi level is significantly higher than the other structures. These high DOS values might favor the superconducting behavior. From the Figure $4(\mathrm{a}-\mathrm{c}), \mathrm{H}$ atoms contribute more to DOS than the Si atoms below Fermi level, and contribute less to DOS than the Si atoms above Fermi level. With increasing pressure, the contributions from atoms $\mathrm{H}$ and $\mathrm{Si}$ do not significantly change. The electron localization functions $(\mathrm{ELF})^{20}$ of the $C 2 / c, P 2_{1} / c$, and $C 2 / m$ phases are calculated at $300 \mathrm{GPa}, 400 \mathrm{GPa}$, and $610 \mathrm{GPa}$, respectively. The isosurface plots at ELF $=0.5$ are shown in Figure $4(\mathrm{~d}-\mathrm{f})$. The electron-gas-like 
Table 1 | Structural parameters for the favored structures suggested in this work

\begin{tabular}{|c|c|c|c|c|c|c|}
\hline \multirow{3}{*}{$\begin{array}{l}\text { Space group \# atoms \# Pressure } \\
P 2_{1} / \mathrm{c} \\
400 \mathrm{GPa}\end{array}$} & \multirow{2}{*}{$\begin{array}{c}\text { Lattice parameters }\left(\AA \AA{ }^{\circ}\right) \\
\qquad a=2.7260\end{array}$} & \multicolumn{4}{|c|}{ Atomic coordinates (fractional) } & \multirow{2}{*}{$\frac{\text { Site }}{4 \mathrm{e}}$} \\
\hline & & $\mathrm{HI}$ & 0.33186 & 0.54676 & 0.91785 & \\
\hline & $b=3.8882$ & $\mathrm{H} 2$ & 0.54601 & 0.83973 & 0.08510 & $4 e$ \\
\hline & $c=4.2525$ & $\mathrm{H} 3$ & 0.65917 & 0.37847 & 0.73239 & $4 e$ \\
\hline & $\beta=88.2231$ & $\mathrm{H} 4$ & 0.17665 & 0.91106 & 0.08146 & $4 e$ \\
\hline & & $\mathrm{Sil}$ & 0.86906 & 0.70548 & 0.84406 & $4 e$ \\
\hline $\mathrm{C} 2 / \mathrm{m}$ & $a=6.7297$ & $\mathrm{HI}$ & 0.52517 & 0.30530 & 0.76854 & $8 \mathrm{j}$ \\
\hline $610 \mathrm{GPa}$ & $b=3.0948$ & $\mathrm{H} 2$ & 0.22275 & 0.19521 & 0.65601 & $8 j$ \\
\hline & $c=6.0792$ & $\mathrm{H} 3$ & 0.22252 & 0.80696 & 0.85411 & $8 j$ \\
\hline & $\beta=63.4329$ & $\mathrm{H} 4$ & 0.87273 & 0.00000 & 0.94004 & $4 \mathrm{i}$ \\
\hline & & H5 & 0.37413 & 0.00000 & 0.68966 & $4 i$ \\
\hline & & H6 & 0.62206 & 0.00000 & 0.81435 & $4 \mathrm{i}$ \\
\hline & & $\mathrm{H} 7$ & 0.11526 & 0.00000 & 0.82576 & $4 \mathrm{i}$ \\
\hline & & $\mathrm{H} 8$ & 0.12208 & 0.00000 & 0.55910 & $4 \mathrm{i}$ \\
\hline & & $\mathrm{H} 9$ & 0.50000 & 0.31851 & 0.00000 & $4 g$ \\
\hline & & $\mathrm{Sil}$ & 0.87715 & 0.00000 & 0.67939 & $4 \grave{i}$ \\
\hline & & $\mathrm{Si} 2$ & 0.61367 & 0.00000 & 0.56500 & $4 \mathrm{i}$ \\
\hline & & $\mathrm{Si} 3$ & 0.37196 & 0.00000 & 0.94522 & $4 \mathrm{i}$ \\
\hline
\end{tabular}

Table 2 | Elastic constants $C_{i j}(\mathrm{GPa})$ of $C 2 / \mathrm{c}, P 2_{1} / \mathrm{c}$, and $C 2 / \mathrm{m}$ crystals calculated at 300,400 , and $610 \mathrm{GPa}$, respectively

\begin{tabular}{|c|c|c|c|c|c|c|c|}
\hline & $C_{11}$ & $C_{22}$ & $C_{33}$ & $C_{44}$ & $C_{55}$ & $C_{66}$ & \multirow[b]{2}{*}{$C_{12}$} \\
\hline & $C_{13}$ & $C_{15}$ & $C_{23}$ & $C_{25}$ & $C_{35}$ & $C_{46}$ & \\
\hline $\mathrm{C} 2 / \mathrm{c}(300 \mathrm{GPa})$ & $\begin{array}{r}1285.47 \\
578.56\end{array}$ & $\begin{array}{r}1117.27 \\
118.06\end{array}$ & $\begin{array}{r}1407.65 \\
796.25\end{array}$ & $\begin{array}{r}408.27 \\
64.00\end{array}$ & $\begin{array}{r}535.20 \\
-146.20\end{array}$ & $\begin{array}{l}242.69 \\
114.85\end{array}$ & 948.40 \\
\hline$P 2_{1} / c(400 \mathrm{GPa})$ & $\begin{array}{r}1590.95 \\
945.00\end{array}$ & $\begin{array}{r}1627.44 \\
34.90\end{array}$ & $\begin{array}{r}1816.35 \\
760.58\end{array}$ & $\begin{array}{l}396.65 \\
-29.57\end{array}$ & $\begin{array}{r}387.36 \\
402\end{array}$ & $\begin{array}{l}224.65 \\
-72.45\end{array}$ & 1072.97 \\
\hline$C 2 / m(610 \mathrm{GPa})$ & $\begin{array}{l}2102.20 \\
1553.58\end{array}$ & $\begin{array}{r}2227.44 \\
-93.95\end{array}$ & $\begin{array}{l}2234.93 \\
1256.96\end{array}$ & $\begin{array}{r}392.92 \\
-50.93\end{array}$ & $\begin{array}{r}483.57 \\
97.95\end{array}$ & $\begin{array}{r}422.65 \\
-2.67\end{array}$ & 1385.08 \\
\hline
\end{tabular}

distribution in this space are connected, which conforms the three structures are metallic.

The electron-phonon coupling strength (EPC) $\lambda$ and the logarithmic average phonon frequency $\omega_{\log }$ of the three structures were calculated to explore the possible superconductivity of $\mathrm{SiH}_{4}$. The Eliashberg phonon spectral function $\alpha^{2} F(\omega)$ and the $\lambda$ as a function of frequency are shown in Figure 5. The $\lambda$ of $C 2 / c$ structure at $300 \mathrm{GPa}, P 2_{1} / c$ structure at $400 \mathrm{GPa}$ and $\mathrm{C} 2 / \mathrm{m}$ structure at $610 \mathrm{GPa}$ are $0.69,0.66$ and 1.18 , respectively. The superconducting critical temperature can be estimated from the Allen-Dynes modified McMillan equation ${ }^{21}$

$$
T_{c}=\frac{\omega_{\log }}{1.2} \exp \left[-\frac{1.04(1+\lambda)}{\lambda-\mu *(1-0.62 \lambda)}\right]
$$

which has been found to be highly accurate for many materials with $\lambda<1.5$. The Coulomb pseudopotential $\mu^{*}$ is taken as 0.13 for hydrogen dominant metallic alloys by Ashcroft ${ }^{22}$, resulting that the estimated $T_{\mathrm{c}}$ of $C 2 / c, P 2_{1} / c$ and $C 2 / m$ at $300 \mathrm{GPa}, 400 \mathrm{GPa}$, and $610 \mathrm{GPa}$ are $29.65 \mathrm{~K}, 31.57 \mathrm{~K}$ and $106.31 \mathrm{~K}$, respectively. Subsequently, the contributions to EPC $\lambda$ of each atom are analyzed. As for $C 2 / c, P 2_{1} / c$ and $C 2 / m$, Si vibrations provide a contribution of $36 \%, 34 \%$, and $37 \%$ respectively, while the $\mathrm{H}$ translational vibrations contribute for nearly $64 \%, 66 \%$ and $63 \%$ respectively. The result shows that the element $\mathrm{H}$ plays a significant role in the EPC $\lambda$.

The trend of $T_{c}$ with pressures for these three structures was explored for further investigation. $T_{c}, \omega_{\log }$ and $\lambda$ along with the changes of pressures are displayed in Figure 6 . With increasing pres-
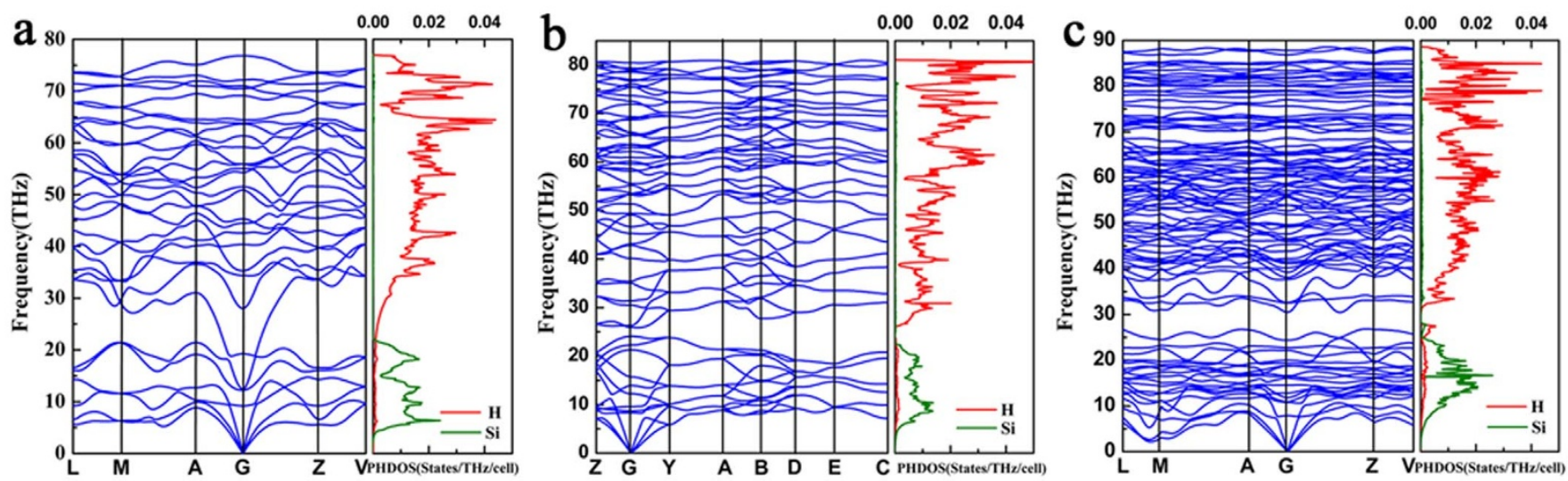

Figure $3 \mid$ The phonon band structure and projected phonon DOS charts. (a) $C 2 / c$ phase at $300 \mathrm{GPa}$. (b) $P 2_{1} / c$ phase at $400 \mathrm{GPa}$. (c) $C 2 / m$ phase at $610 \mathrm{GPa}$ 


\section{a}

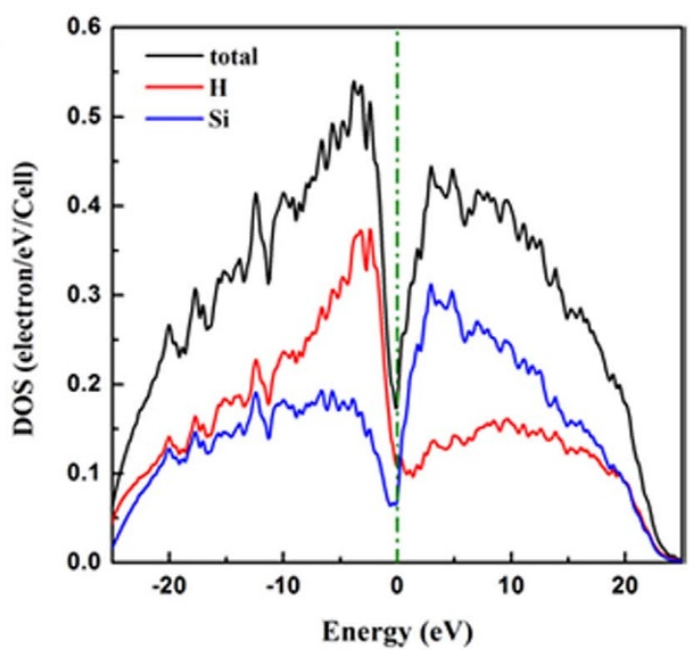

b

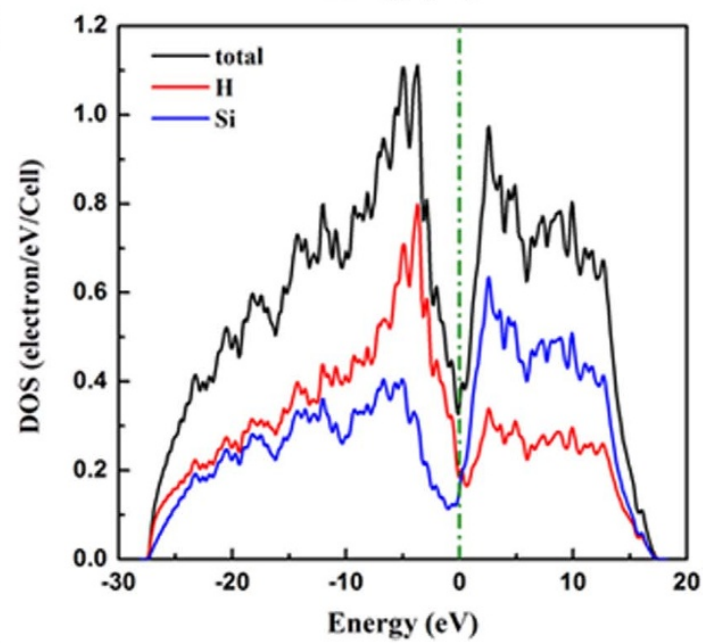

C

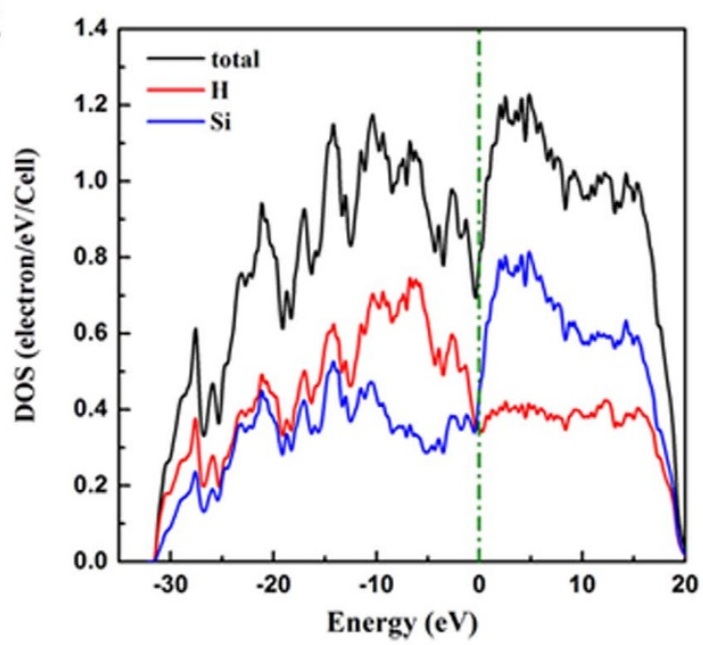

d

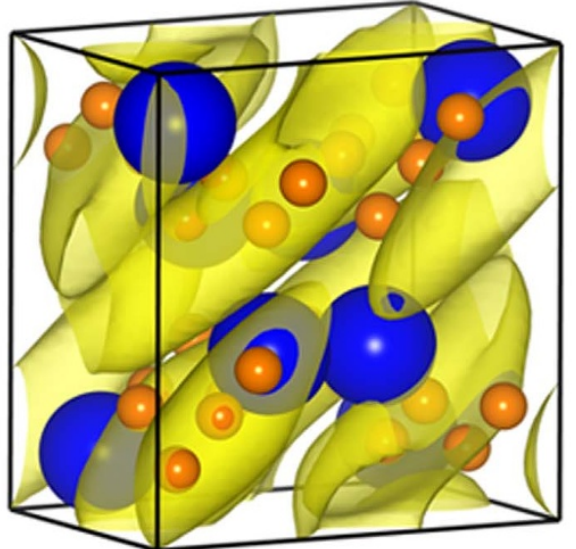

e

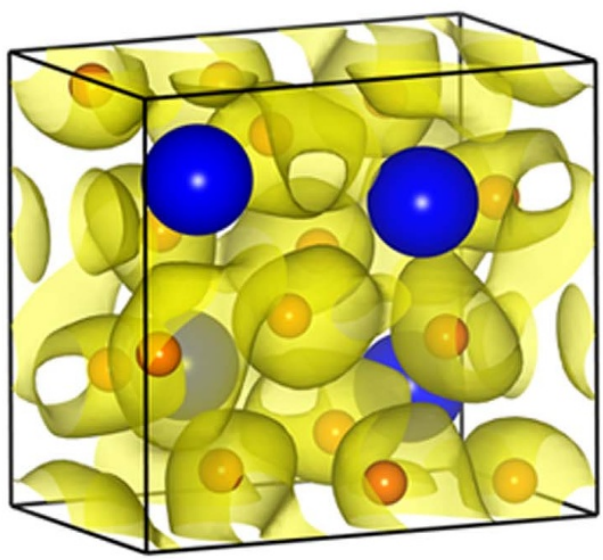

$\mathrm{f}$

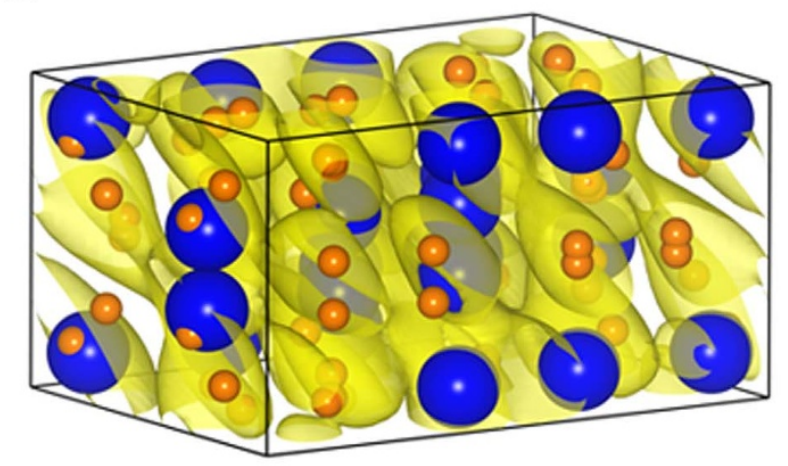

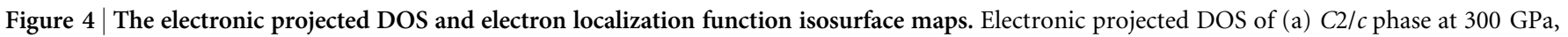

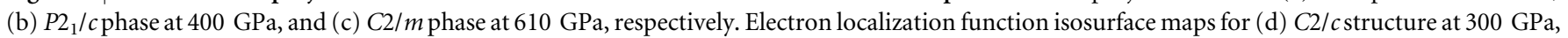
(e) $P 2_{1} / c$ structure at $400 \mathrm{GPa},(\mathrm{f}) \mathrm{C2} / \mathrm{m}$ structure at $610 \mathrm{GPa}$. The yellow isosurface represents an ELF value of 0.5 . Blue and orange spheres represent $\mathrm{Si}$ and $\mathrm{H}$, respectively.

sure, the decrease of $\lambda$ plays an important role to the downward trend of $T_{\mathrm{c}}$. Similar phenomena can be observed in other hydrogen-rich materials ${ }^{10,23-25}$. We then use the rigid-muffin-tin (RMT) theory of Gaspari and Gyorffy ${ }^{26}$ for further analysis this variation. MaMillan's strong coupling theory ${ }^{22,27}$ defines an electron-phonon coupling constant by

$$
\lambda=\frac{\eta}{M\left\langle\omega^{2}\right\rangle^{\frac{1}{2}}}=\frac{N\left(\varepsilon_{F}\right)\left\langle I^{2}\right\rangle}{M\left\langle\omega^{2}\right\rangle^{\frac{1}{2}}},
$$

where $M$ is the atomic mass. The DOS at the Fermi level $N\left(\epsilon_{F}\right)$, the square of the electron-ion matrix element $\left\langle I^{2}\right\rangle$ and the average pho- 


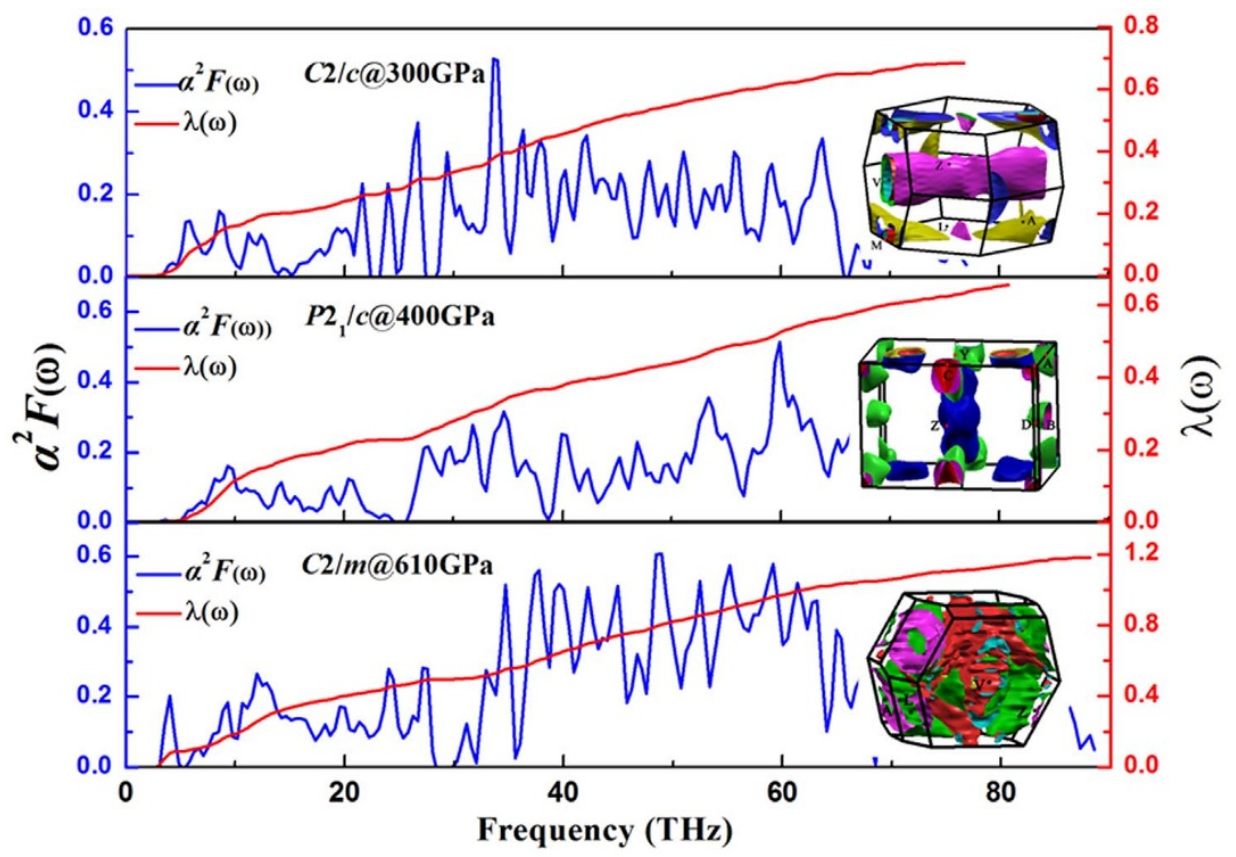

Figure $5 \mid$ The Eliashberg phonon spectral function $\alpha^{2} F(\omega)$, the electron-phonon integral $\lambda$ and the three-dimensional Fermi surface of $C 2 / c, P 2 / c$ and $C 2 / m$ at 300,400 , and $610 \mathrm{GPa}$, respectively.

non frequency $\left\langle\omega^{2}\right\rangle^{\frac{1}{2}}$ of the three phases at several pressures are shown in Figure 6. From the Figure, $\left\langle\omega^{2}\right\rangle^{\frac{1}{2}}$ increases generally with pressure, meanwhile $N\left(\epsilon_{F}\right)$ decrease (the values between $C 2 / m$ at $610 \mathrm{GPa}$ and $700 \mathrm{GPa}$ are closely identical). The tendency of the two parameters made $\lambda$ become lower and lower, which lead to the decrease of $T_{c}$ with increasing pressure.

We also realized that $T_{\mathrm{c}}$ magnitude of $C 2 / \mathrm{m}$ is beyond $10^{2} \mathrm{~K}$ which is higher than other two phases that promoted us to explore the underlying superconducting mechanism. From Figure 6, the

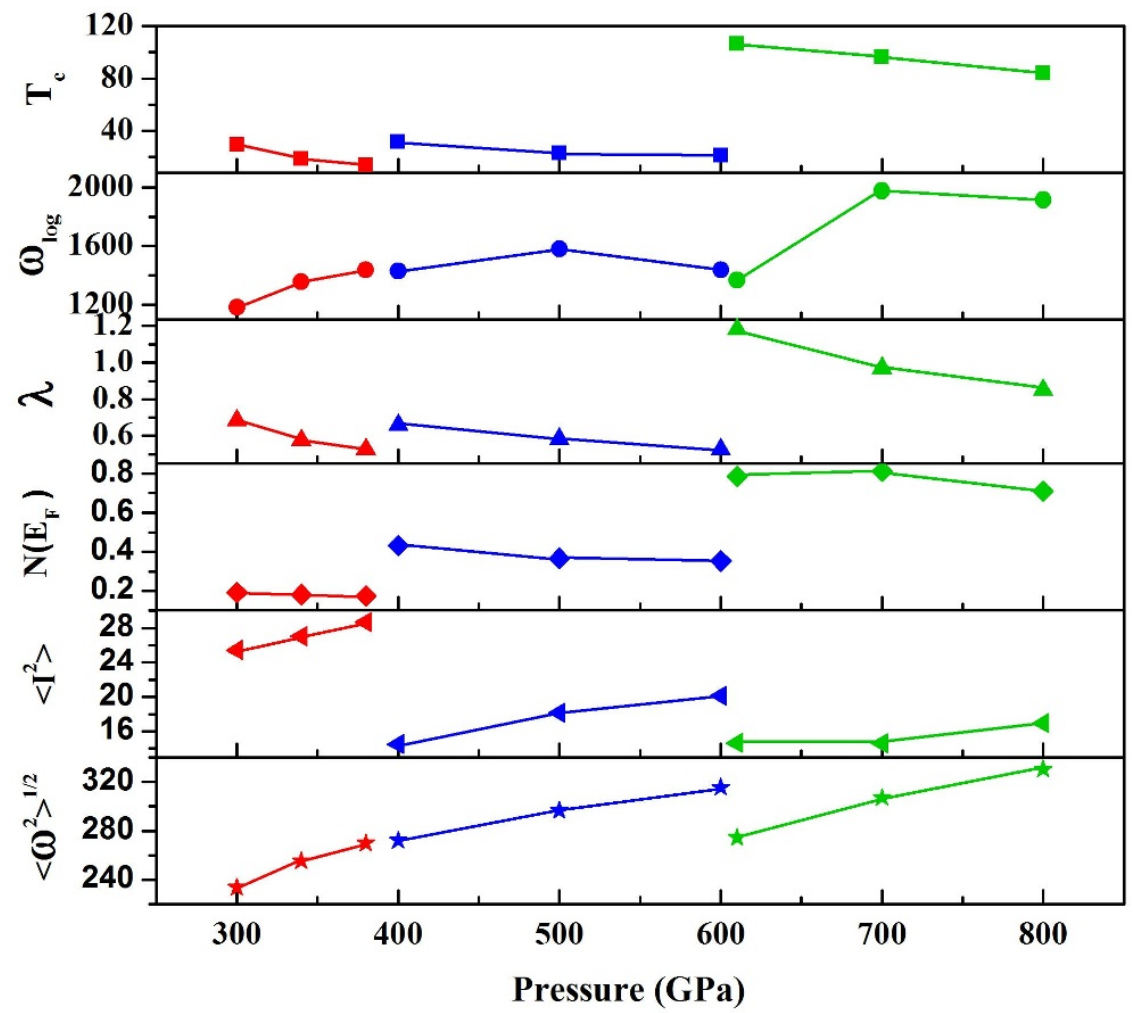

Figure $6 \mid$ Calculated $T c(\mathrm{~K})$, the logarithmic average phonon frequency $\omega_{\log }(\mathrm{K})$, electron-phonon coupling strength $\lambda$, the DOS at the Fermi level $\mathrm{N}\left(\epsilon_{\mathrm{F}}\right)\left(\right.$ state/eV/Cell), the square of the electron-ion matrix element $\left\langle I^{2}\right\rangle(\mathrm{eV} / \AA)^{2}$ and the average phonon frequency $\left\langle\omega^{2}\right\rangle^{\frac{1}{2}}(\mathrm{THz})$ of the three phases as a function of pressure. The red line respect $C 2 / c$ phase at 300-380 GPa. The blue line respect $P 2_{1} / c$ phase at $400-600 \mathrm{GPa}$. The green line respect $C 2 / m$ phase at $610-800 \mathrm{GPa}$. 
trend of $\lambda$ along with pressures is consistent with $T_{\mathrm{c}}$. So, $\lambda$ played an important role in the superconducting critical temperature. The calculated EPC $\lambda$ of $C 2 / m$ phase at $610 \mathrm{GPa}$ is 1.18 , which is much higher than the values of 0.69 and 0.66 of two phases. The bigger EPC $\lambda$ can directly contribute to higher $T_{\mathrm{c}}$. Furthermore, formula (2) defined by MaMillan's strong coupling theory can be used to analyze the mechanisms. Comparing the contribution to $\lambda$ in the phase of $C 2 / \mathrm{m}$ and $C 2 / \mathrm{c}$, Figure 6 reveals that $N\left(\epsilon_{F}\right)$ of $C 2 / \mathrm{m}$ contributes about four times than the one of $C 2 / c$, meanwhile the two factors of $\left\langle\omega^{2}\right\rangle^{\frac{1}{2}}$ and $\left\langle I^{2}\right\rangle$ together contribute about half of $C 2 / c$. Although there are two disadvantages of parameters in $C 2 / m$ according formula (2), the higher $N\left(\epsilon_{F}\right)$ sustains the higher $\lambda$ as a whole. As for $C 2 / m$ and $P 2_{1} / c$, there are no obvious differences on the values of $\left\langle\omega^{2}\right\rangle^{\frac{1}{2}}$ and $\left\langle I^{2}\right\rangle$, and $\lambda$ can be dominated by $N\left(\epsilon_{F}\right)$ as well. As shown in Figure 6, $N\left(\epsilon_{F}\right)$ in $C 2 / m$ is much higher than the ones in other two structures. Based on an overall analysis of contributions from parameters in two formulas including the contribution from $\omega_{\log }, N\left(\epsilon_{F}\right)$ play an important role to the much higher $T_{c}$ than other phases.

It is consistent with the calculated three-dimensional Fermi surface which is shown in Figure 5. There are four electronic bands across the Fermi surface for all the three phases, and identified by different colors. As shown in Figure 5, the $C 2 / m$ phase is filling with much more Fermi surfaces in the brillouin zone than the two other phases, which is corresponding with the high level of $N\left(\epsilon_{F}\right)$. Therefore, the Fermi surface also support the conclusion that the high values of $N\left(\epsilon_{F}\right)$ is significant to sustain high $T_{c}$.

\section{Conclusion}

In summary, we have extensively investigated crystal structures and superconductivity of silane. The $P 2_{1} / c$, and $C 2 / m$ structures are found which are thermodynamically, mechanically, and dynamically stable. The superconducting critical temperature $T_{\mathrm{c}}$ of the $C 2 / c$ phase at $300 \mathrm{GPa}$ and $P 2_{1} / c$ at $400 \mathrm{GPa}$ are $29.65 \mathrm{~K}$ and $31.57 \mathrm{~K}$. The superconductivity of the $C 2 / \mathrm{m}$ phase with a transition temperature $106.31 \mathrm{~K}$ is found to be mainly attributed to the strong electronphonon coupling due to the high electronic density of states at the Fermi level.

\section{Methods}

The most stable structures of silane under high pressures were performed using evolutionary algorithm, as implemented in the USPEX code ${ }^{28}$. This approach has been successfully used in the study of many materials at high pressures ${ }^{29,30}$. The structural relaxations have been performed within the framework of the generalized gradient approximation (GGA) ${ }^{31}$ with the Perdew-Burke-Ernzerhof parameterization for the exchange-correlation functional to DFT by using the projector augmented-wave(PAW) method ${ }^{32}$, as implemented in $a b$ initio VASP code ${ }^{33}$. The structures were relaxed at a high cutoff energy of $1000 \mathrm{eV}$, and a Brillouin zone sampling grid of spacing $2 \pi \times 0.03 \AA^{-1}$. The lattice dynamics and electron-phonon coupling have been computed with QUANTUM-ESPRESSO ${ }^{34}$. We used Vanderbilttype ultrasoft pseudopotentials ${ }^{35}$ with a cutoff energy of 40 Ry. Phonon frequencies were calculated based on the density functional linear-response method ${ }^{36}$. A Monkhorst-Pack(MP) $)^{37}$ Brillouin zone sampling grid of spacing $2 \pi \times 0.025 \AA^{-1}$ with Gaussian smearing of 0.02 Ry were used for the phonon calculations, at $4 \times 4 \times 4,5$ $\times 4 \times 3$ and $3 \times 3 \times 2 q$-point mesh for $C 2 / c, P 2_{1} / c$ and $C 2 / m$ for the electronphonon interaction matrix element, respectively. All the convergences of the planewave basis set and MP sampling are carefully examined by employing higher kinetic energy cutoffs and denser grids sets. The tests of cutoff energy and the validity of potential functions are shown in supplementary information.

1. Ashcroft, N. W. Metallic hydrogen: a high-temperature superconductor? Phys. Rev. Lett. 21, 1748 (1968).

2. Cudazzo, P. et al. Ab initio description of high-temperature superconductivity in dense molecular hydrogen. Phys. Rev. Lett. 100, 257001 (2008).

3. Loubeyre, P., Occelli, F. \& LeToullec, R. Optical studies of solid hydrogen to $320 \mathrm{GPa}$ and evidence for black hydrogen. Nature 416, 613-617 (2002).

4. Goncharov, A. F., Gregoryanz, E., Hemley, R. J. \& Mao, H. Spectroscopic studies of the vibrational and electronic properties of solid hydrogen to $285 \mathrm{GPa}$. Proc. Natl. Acad. Sci. U.S.A. 98, 14234-14237 (2001).
5. Gao, G. et al. Superconducting high pressure phase of germane. Phys. Rev. Lett. 101, 107002 (2008)

6. Gao, G. et al. High-pressure crystal structures and superconductivity of stannane $\left(\mathrm{SnH}_{4}\right)$. Proc. Natl. Acad. Sci. U.S.A. 107, 1317-1320 (2010).

7. Feng, J. et al. Structures and potential superconductivity in $\mathrm{SiH}_{4}$ at high pressure: en route to "metallic hydrogen" Phys. Rev. Lett. 96, 017006 (2006).

8. Pickard, C. J. \& Needs, R. J. High-pressure phases of silane. Phys. Rev. Lett. 97 045504 (2006).

9. Yao, Y., Tse, J. S., Ma, Y. \& Tanaka, K. Superconductivity in high-pressure $\mathrm{SiH}_{4}$ Euro. Phys. Lett. 78, 37003 (2007)

10. Eremets, M. I., Trojan, I. A., Medvedev, S. A., Tse, J.S. \& Yao, Y. Superconductivity in hydrogen dominant materials: silane. Science 319, 1506-1509 (2008).

11. Chen, X. et al. Superconducting behavior in compressed solid $\mathrm{SiH} 4$ with a layered structure. Phys. Rev. Lett. 101, 077002 (2008).

12. Martinez-Canales, M. et al. Novel structures and superconductivity of silane under pressure. Phys. Rev. Lett. 102, 087005 (2009).

13. Pickard, C. J. \& Needs, R. J. Structure of phase III of solid hydrogen. Nat. Phys. 3, 473-476 (2007).

14. Degtyareva, O. et al. Crystal structure of SiH4 at high pressure. Phys. Rev. B 76, 064123 (2007).

15. Johnson, K. A. \& Ashcroft, N. W. Structure and bandgap closure in dense hydrogen. Nature 403, 632-634 (2000).

16. Katzke, H., Bismayer, U. \& Tolédano, P. Theory of the high-pressure structural phase transitions in Si, Ge, Sn, and Pb. Phys. Rev. B 73, 134105 (2006).

17. Jin, X. et al. Superconducting high-pressure phases of disilane. Proc. Natl. Acad. Sci. U.S.A. 107, 9969-9973 (2010).

18. Nye, N. F. Physical Properties Of Crystal. 142-143 (Oxford Univ. Press, Oxford, 1985).

19. Wu, Z. et al. Crystal structures and elastic properties of superhard $\operatorname{IrN}_{2}$ and $\operatorname{IrN}_{3}$ from first principles. Phys. Rev. B 76, 054115 (2007).

20. Becke, A. D. \& Edgecombe, K. E. A simple measure of electron localization in atomic and molecular systems. J. Chem. Phys. 92, 5397-5403 (1990).

21. Allen, P. B. \& Dynes, R. C. Transition temperature of strong-coupled superconductors reanalyzed. Phys. Rev. B 12, 905-922 (1975).

22. Ashcroft, N. W. Hydrogen Dominant metallic alloys: high temperature superconductors? Phys. Rev. Lett. 92, 187002 (2004).

23. Flores-Livas, J. A. et al. High-Pressure Structure of Disilane and Their Superconducting Properties. Phys. Rev. Lett. 108, 117004 (2012).

24. Kim, D. K. et al. Predicted Formation of Superconducting Platinum-Hydride Crystals under Pressure in the Presence of Molecular Hydrogen. Phys. Rev. Lett. 107, 117002 (2011).

25. Zhou, X. F. et al. Superconducting high-pressure phase of platinum hydride from first principle. Phys. Rev. B 84, 054543 (2011).

26. Gaspari, G. D. \& Gyorffy, B. L. Electron-Phonon Interactions, $d$ Resonances, and Superconductivity in Transition Metals. Phys. Rev. Lett. 28, 801-805 (1972).

27. McMillan, W. L. Transition Temperature of Strong-Coupled Superconductors. Phys. Rev. 167, 331-344 (1968).

28. Oganov, A. R. \& Glass, C. W. Crystal structure prediction using $a b$ initio evolutionary techniques: principles and applications. J. Chem. Phys. 124, 244704 (2006).

29. Zhu, Q. et al. Stability of xenon oxides at high pressure. Nat. Chem. 5, 61-65 (2013).

30. Zhang, W. et al. Unexpected stable stoichiometries of sodium chlorides. Science 342, 1502 (2013)

31. Perdew, J. P., Burke, K. \& Ernzerhof, M. Generalized gradient approximation made sample. Phys. Rev. Lett. 77, 3865-3858 (1996).

32. Kresse, G. \& Joubert, D. From ultrasoftpseudopotentials to the projector augmented-wave method. Phys. Rev. B 59, 1758-1775 (1999).

33. Kresse, G. \& Furthmüller, J. Efficient iterative schemes for ab initio total-energy calculations using a plane-wave basis set. Phys. Rev. B 54, 11169-11186 (1996).

34. Giannozzi, P. et al. QUANTUM ESPRESSO: a modular and open-source software project for quantum simulations of materials. J. Phys. : Condens. Matter 21, 395502 (2009).

35. Vanderbilt, D. Soft self-consistent pseudopotentials in a generalized formalism. Phys. Rev. B 41, 7892-7895 (1990).

36. Baroni, S., Giannozzi, P. \& Testa, A. Green's-function approach to linear response in solids. Phys. Rev. Lett. 58, 1861-1864 (1987).

37. Monkhorst, H. J. \& Pack, J. D. Special points for Brillouin-zone integrations. Phys. Rev. B 13, 5188-5192 (1976).

\section{Acknowledgments}

This work was supported by the National Basic Research Program of China (No. 2011CB808200), Program for Changjiang Scholars and Innovative Research Team in University (No. IRT1132), the National Natural Science Foundation of China (Nos. 51032001, 11074090, 10979001, 51025206, 11174102), and National Found for Fostering Talents of basic Science (No. J1103202). Parts of calculations are performed at the High Performance Computing Center (HPCC) of Jilin University. 


\section{Author contributions}

T.C. initiated the project. H.Z. performed the first principle calculations and prepared all figures. H.Z., X.J. and T.C. analyzed the data and wrote the manuscript text. Y.Z.L., Q.Z., Y.X.L., Q.L., K.B., D.L. and B.L. reviewed the manuscript.

\section{Additional information}

Supplementary information accompanies this paper at http://www.nature.com/ scientificreports

Competing financial interests: The authors declare no competing financial interests.
How to cite this article: Zhang, H. et al. High-temperature Superconductivity in compressed Solid Silane. Sci. Rep. 5, 8845; DOI:10.1038/srep08845 (2015).

This work is licensed under a Creative Commons Attribution 4.0 International License. The images or other third party material in this article are included in the article's Creative Commons license, unless indicated otherwise in the credit line; if the material is not included under the Creative Commons license, users will need to obtain permission from the license holder in order to reproduce the material. To view a copy of this license, visit http://creativecommons.org/licenses/by/4.0/ 